\title{
White Matter Integrity Correlates of the Reading Span
}

\author{
Ekaterina V. Pechenkova ${ }^{1}$, Yana R. Panikratova ${ }^{2}$, Maria A. Fomina ${ }^{3}$, Elena A. Mershina ${ }^{4}$, \\ Daria A. Bazhenova ${ }^{4}$, Liudmila A. Makovskaya ${ }^{5}$, Alena D. Rumshiskaya ${ }^{6}$, Olga V. \\ Fedorova $^{7}$, Valentin E. Sinitsyn ${ }^{4}$, Irina S. Lebedeva ${ }^{2}$, Roza M. Vlasova $^{8}$
}

${ }^{1}$ Laboratory for Cognitive Research, Higher School of Economics, Moscow, Russia Correspondence: 4 Armyanskiy ln., bld. 2, 101000 Moscow Russia; evp@virtualcoglab.org ${ }^{2}$ Laboratory of Neuroimaging and Multimodal Analysis, Mental Health Research Center, Moscow, Russia

${ }^{3}$ Faculty of Psychology, Lomonosov Moscow State University, Moscow, Russia

${ }^{4}$ Medical Research and Educational Center (Lomonosov University Clinic), Moscow, Russia

${ }^{5}$ Radiology Department, Federal Center of Treatment and Rehabilitation, Moscow, Russia

${ }^{6}$ Radiology Department, Davidovskiy City Clinical Hospital, Moscow, Russia

${ }^{7}$ Faculty of Philology, Lomonosov Moscow State University, Moscow, Russia

${ }^{8}$ Department of Psychiatry, University of North Carolina at Chapel Hill, USA

\begin{abstract}
Although working memory (WM) is crucial for intellectual abilities, not much is known about its brain underpinnings, especially the structural connectivity. We used diffusion tensor imaging (DTI) to look across the whole brain for the white matter integrity correlates of the individual differences in the reading span (verbal WM capacity during reading) in healthy adults. Right-handed healthy native Russian speakers $(\mathrm{N}=67)$ underwent DTI on a 3T Philips Ingenia scanner. Verbal WM was assessed with the Daneman-Carpenter reading span test (Russian version). Fractional anisotropy maps from each participant were entered into the group tract-based spatial statistics analysis with the reading span as a covariate; the results were TFCE-corrected. After taking into account effects of age, sex, education and handedness, reading span positively correlated with the white matter integrity in multiple sites: the body, the genu and the splenium of corpus callosum; bilateral corona radiata (anterior, posterior, and superior); bilateral superior longitudinal fasciculus; several tracts in the right hemisphere only, including the internal and external capsule; bilateral superior parietal and frontal white matter. Although the left hemisphere is central for verbal processing, we revealed the important role of the right hemisphere white matter for the verbal WM capacity. Our finding indicates that larger verbal working memory span may originate from additional processing resources of the right hemisphere.
\end{abstract}

Keywords: working memory; reading span; white matter; DTI 


\section{Introduction}

Working memory is a limited capacity system for short-term information storage and manipulation which lies in the core of human cognitive and executive functions. It is crucial for learning and intellectual abilities (Cowan, 2014; Süß et al., 2002; Oberauer et al., 2005), and many neurological conditions and developmental disorders are characterized by working memory deficits (Goldman-Rakic, 1994; Sandry, 2015; Alloway et al., 2009).

Working memory models stem from the multiple memory systems approach which suggests that memory is composed of at least two subsystems, a short-term and a long-term memory. Extending the idea of short-term memory, working memory concept implies that information is not only maintained within a temporary storage system, but is also actively manipulated to complete the cognitive task at hand (Miller et al., 1960; Baddeley and Hitch, 1974; Cowan, 2008). Multicomponent model of the working memory, proposed and elaborated by Baddeley and Hitch (Baddeley \& Hitch, 1974; Baddeley, 2001, 2003, 2012) includes specific storages for phonological and visuospatial formats along with the central executive component operating on representations in different formats. This approach received support from individual difference studies which revealed a corresponding threefactor structure of the working memory abilities across different age groups (Alloway et al., 2004; Gathercole et al., 2004; Hornung, Brunner, Reuter, \& Martin, 2011). In assessment of individual capacities of storages for phonological and visuospatial formats a distinction between verbal and non-verbal working memory is widely used (Baddeley, 2003).

Being the most influential, Baddeley's multicomponent model is not the only. Questions of whether working memory is a separate memory subsystem or an activated part of the long-term memory (Cowan, 1988, 1999; Oberauer, 2002) and on specific cognitive and executive mechanisms within the working memory such as information maintenance, updating or protection from interference have been highly debated for several decades (Miyake, Friedman, Emerson, Witzki, Howerter, \& Wager, 2000; Engle, 2002; Ecker, Lewandowsky, Oberauer, \& Chee, 2010; Schmiedek, Lövdén, \& Lindenberger, 2014). In accordance with these different theoretical perspectives a variety of working memory 
capacity measures were developed, tapping verbal and non-verbal information storage and processing and including simple span tasks (immediate recall of a list of words, digits, letters or object spatial locations), complex span tasks (dual-task requiring that items are maintained in memory during a concurrent cognitive activity such as reading), updating tasks, such as the n-back task (comparing the present item in a continuously updating sequence with an item presented $\mathrm{n}$ steps back), and some other (see Wilhelm et al., 2013 for a review). While simple spans are widely adopted in cognitive ability test batteries (Wechsler scales, AWMA, CANTAB), complex spans have shown to better predict real-life cognitive tasks performance. The reading span test (Daneman \& Carpenter, 1980) which was originally developed as a proof of concept for the complex span tasks, was found to predict reading comprehension much better than word span or digit span tasks addressing mostly short-term storage of the verbal material (Daneman \& Merikle, 1996) and is nowadays widely used in psycholinguistics.

Neural underpinnings of the working memory are not yet described in such detail as its behavioral aspects. Neurocognitive models have shown importance of both cortical and subcortical structures for different working memory processes and components, including language areas of the left hemisphere; visual cortices; fronto-parietal network, which recruits the dorsolateral prefrontal cortex, parietal cortex, and anterior cingulate; and even basal ganglia (see Chai et al., 2018 for review). As these models are built mostly on the functional neuroimaging data, they describe predominantly the grey matter impact, while underlying structural connectivity may also be of great importance.

Existing diffusion tensor imaging (DTI) studies of the working memory address different populations from infants to ageing, both healthy and clinical samples. Overall DTI data indicate that working memory implicates white matter integrity across almost entire brain in infancy (Short et al., 2013), while later the most frequent findings are located in fronto-parietal pathways (superior and inferior longitudinal fasciculi), posterior temporal, and parietal white matter, as well as in corpus callosum, and are more pronounced in the left hemisphere (Bathelt et al., 2018; Chung et al., 2018; Darki \& Klingberg, 2015; Klingberg, 
2006; Krogsrud et al., 2018; Olesen et al., 2003; Schulze et al., 2011). Bathelt et al. (2018) presented the only attempt to assess the white matter integrity correlates for the visuo-spatial (non-verbal), verbal and executive components of the working memory measured with AWMA in the same group of children. In most research, working memory was assessed with either n-back task (updating; Schulze et al., 2011; Takeuchi et al., 2010; Burzynska et al., 2011; Sala-Llonch et al 2015; Salminen et al. 2016; Takahashi et al., 2010) or with simple span tasks: verbal, such as digit or letter span (Chung et al., 2018; Takeuchi et al., 2011; Ekman et al., 2016; Krogsrud et al., 2018; Zahr et al., 2009; Charlton et al., 2013; Østby et al., 2011) or nonverbal, such as spatial span or Corsi block (Nagy et al., 2004; Zahr et al., 2009; Krogsrud et al., 2018; Vestergaard et al., 2011; Takeuchi et al., 2010; Darki \& Klingberg, 2015; Olesen et al., 2003).

Therefore white matter tract-based correlates of the complex span working memory performance remain unknown, although they may much better describe the neural substrates of working memory functioning in the real-life cognitive behavior such as reading and writing, and better predict academic and professional success in language-related spheres. To fill this gap, we conducted a tract-based spatial statistics (TBSS) study in the healthy adult population with a focus on reading span which characterizes the verbal working memory capacity during reading (Daneman, Carpenter, 1980). We used diffusion tensor imaging (DTI) to obtain the fractional anisotropy (FA) measures for each voxel across the whole brain which were further correlated with the individual differences in the reading span measured outside of the scanner.

\section{Methods}

\section{Participants}

Ninety two healthy volunteers from Moscow, Russia (35 males, 57 females, aged $24.4 \pm 5.6$ y.o., mean years of education $14.9 \pm 2.6$ ) took part in the study. The following inclusion criteria were used: native speaker of Russian; right-handed (handedness was assessed with laterality quotient 10 [LQ10] from Edinburgh Handedness Inventory; Oldfield, 
1971); no contraindications for the MRI procedures (assessed with a screening questionnaire); no reported history of neurological or mental conditions. The project was approved by the Interuniversity Ethics Committee of Moscow. Participants gave written informed consent to the MRI and psychological assessment and received financial compensation for their time and effort.

Data from 2 participants were excluded from the analysis due to technical issues (wrong imaging protocol or signal loss induced by metallic dental implant), from 2 more participants due to distraction from neuropsychological tasks, and from 21 due to substantial checkerboard artifact (Oguz et al., 2014) revealed by radiologists during visual inspection of their DWI images. Therefore data analysis was performed on a subset of 67 participants (23 males, 44 females, mean age $24.0 \pm 5.3$ y.o, mean years of education $15.1 \pm 2.5$, handedness: LQ10 range 60-100, median 100).

\section{Materials}

Reading span (verbal working memory) was assessed with the Russian version (Fedorova, 2010) of the Daneman-Carpenter test (Daneman, Carpenter, 1980). Participants were tested individually in a quiet room. They were shown sentences via PowerPoint presentation on a laptop, one sentence per slide. The task was to read each sentence aloud and immediately proceed to the next slide. Time per slide was not limited in a way other than participant's reading speed. After a set of a few sentences, a blank slide appeared, and participants were asked to recall the last word of each sentence from the set they just have read in the correct order and grammatical form (gender, number, case, and tense). All responses were audio recorded. Overall the test contained 20 sets of sentences, from 2 up to 5 per set, 5 sets of each size (i.e., 5 sets of 2 sentences, 5 sets of 3 sentences etc.). Performance was measured as a percentage of correctly recalled groups (without any omission or grammatical and ordinal error), $5 \%$ per set. 


\section{Neuroimaging}

MR images were acquired on a 3T Philips Ingenia MRI scanner located at the Mental Health Research Center, Moscow, Russia. The DTI data were obtained with a 15-channel dStream HeadSpine coil and standard Philips single-shot EPI sequence with the following parameters: $\mathrm{TR}=3951 \mathrm{~ms}, \mathrm{TE}=96 \mathrm{~ms}$, flip angle $90^{\circ}$, SENSE parallel imaging acceleration (factor of 2), field of view $256 \times 256 \times 138 \mathrm{~mm}$, imaging matrix $112 \times 112,60$ near-transverse slices with isotropic voxel size $2.3 \mathrm{~mm}$, ascending interleaved slice acquisition order; diffusion-weighted images: $\mathrm{b}=1000 \mathrm{~s} / \mathrm{mm}^{2}, 32$ diffusion directions with AP phase encoding direction and 6 diffusion directions with PA phase encoding direction; diffusion directions were distributed across a half-sphere (standard Philips settings). A pair of T2-weighted images $\left(b=0 \mathrm{~s} / \mathrm{mm}^{2}\right.$ ) with the opposite phase encoding directions (AP and PA) was also acquired. The DTI data were complemented with the T1-weighted structural images acquired with the TFE sequence $\left(\mathrm{TR} / \mathrm{TE}=8 \mathrm{~ms} / 4 \mathrm{~ms}\right.$, flip angle $8^{\circ}, 170$ near-transverse slices, isotropic voxel size $1 \mathrm{~mm})$.

\section{Procedure}

The DWI was performed in either the beginning or end of the one hour and a half neuroimaging session which included data collection for a speech perception study. The working memory assessment (reading span test) was administered either before or after the scanning session along with other behavioral tests.

\section{Data Analysis}

As a first step of the DTI data processing, two radiologists (L.M. and D.B.) performed a visual screening of the T1-weighted images for anatomy anomalies potentially critical for the present study and DWI data for image artifacts while being blind of the behavioral test results of the participants. Then an automated quality check procedure was applied with the DTIPrep software (Oguz et al., 2014) to detect volumes corrupted by excessive head motion or intensity abnormalities. Volumes affected by any data quality issue were removed from 
further processing; there were no subjects for whom the portion of the remaining DWI data was less than $75 \%$.

Further processing was performed with FSL software (Jenkinson et al., 2012; http://fsl.fmrib.ox.ac.uk/fsl/fslwiki/) and included correction of the eddy currents (eddy_correct), correction of the metric distortions with a fieldmap calculated from non-DW images with the opposite phase encoding direction (topup and applytopup), skull stripping of all data and spatial coregistration of the DTI and structural images. Diffusion tensor model was fitted voxelwise on individual participant's preprocessed data, and fractional anisotropy (FA) maps were computed.

Using the FSL Tract-Based Spatial Statistics (TBSS) workflow (Smith et al., 2006), FA individual maps were then registered to the common FA image $1 \mathrm{~mm}$ template in MNI space and a group white matter skeleton was created from FA maps; individual diffusion tensor imaging (DTI) metrics were projected onto the skeleton. One-sample $t$-tests with the mean-centered reading span score as a covariate of interest were performed on skeletonized FA images using nonparametric permutation inference (Winkler et al., 2014); mean-centered age, sex, years of education and handedness (laterality quotient) were included into the model as covariates of no interest to account for extra FA variability. The number of permutations was set to 5000 and the results were corrected for multiple comparisons using the thresholdfree cluster enhancement method (TFCE) with $p<0.05$. The anatomical localization of significant clusters was defined with ICBM-DTI-81 white-matter labels probabilistic atlas (https://fsl.fmrib.ox.ac.uk/fsl/fslwiki/Atlases). For better visualization of the results on the skeleton (Figure 1), we thickened the thresholded $p$-value image using tbss_fill function. 


\section{Results}

Reading span scores ranged from 20 to $95 \%$ (mean $45 \pm 15 \%$ ). After taking into account effects of sex, age, education and handedness, reading span positively correlated with the white matter integrity in multiple sites: the body, the genu and the splenium of corpus callosum; bilateral anterior, posterior, and superior corona radiata; bilateral superior longitudinal fasciculus; cerebral peduncle, anterior and posterior limb of the internal capsule, retrolenticular part of internal capsule, posterior thalamic radiation, sagittal stratum, external capsule, and fornix in the right hemisphere; bilateral parietal and frontal white matter outside the scope of the atlas (see Figure 1).

\section{Discussion}

The present study has revealed that the verbal working memory capacity in real-life cognitive activity, such as reading, is associated with the integrity of extensive portions of the white matter tracts in both hemispheres. Implication of the corpus callosum, corona radiata, and superior longitudinal fasciculi in working memory capacity is consistent with the earlier DTI studies on different populations and other working memory measures, both verbal and non-verbal (Bathelt et al., 2017; Chung et al., 2018; Darki \& Klingberg, 2015). At the same time, unlike most of the previously reported data, our results indicate bilateral nature of the verbal working memory capacity neural substrate, even with a right-sided lateralization of the white matter integrity correlates in several structures. Noteworthy, Takeuchi et al. (2011) have previously reported right-sided parietal white matter integrity correlates of an index reflecting information processing component in the verbal working memory. Our finding indicates that larger verbal working memory span may originate from additional processing resources of the right hemisphere. Looking for potential white-matter right-hemisphere correlates of the verbal working memory deficits seems to be a promising direction of future research. 


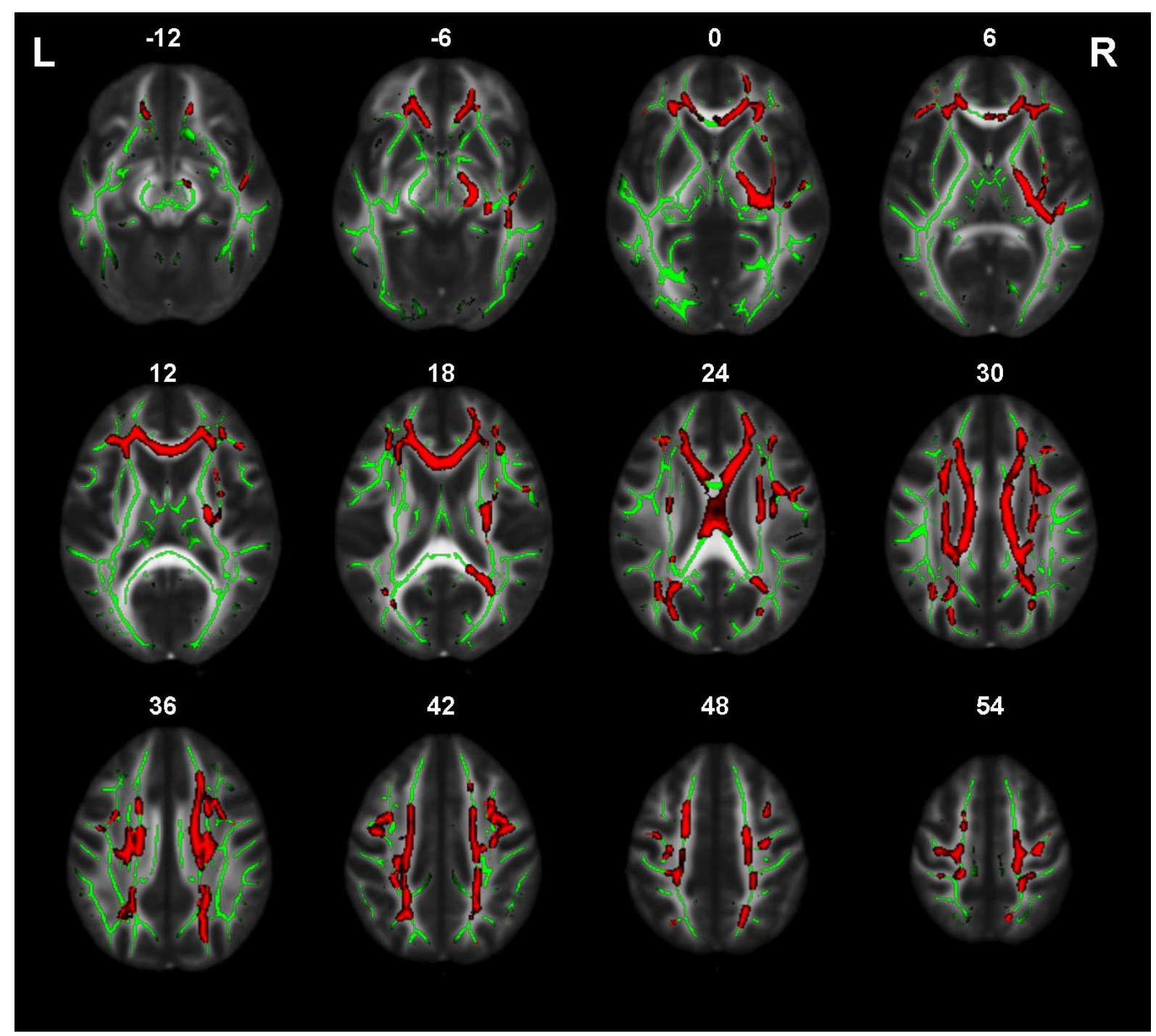

Figure 1. Parts of the white matter skeleton that were significantly associated with working memory capacity (reading span) after taking into account effects of age, sex, education and handedness, threshold-free cluster enhancement method (TFCE) with $p<0.05$. Skeleton is shown in green. Results are overlaid on the target FA image. Slice indices represent MNI coordinates (z).

\section{Acknowledgements}

The study was supported by RFBR grant 18-00-01598(18-00-01592). 


\section{References}

Alloway, T. P., Gathercole, S. E., Willis, C., \& Adams, A. M. (2004). A structural analysis of working memory and related cognitive skills in young children. J Exp Child Psychol, 87(2), 85-106. doi: 10.1016/j.jecp.2003.10.002

Alloway, T. P., Rajendran, G., \& Archibald, L. M. (2009). Working memory in children with developmental disorders. J Learn Disabil, 42(4), 372-382. doi: $10.1177 / 0022219409335214$

Baddeley, A. (2003). Working memory: looking back and looking forward. Nat Rev Neurosci, 4(10), 829-839. doi: 10.1038/nrn1201

Baddeley, A. (2012). Working memory: theories, models, and controversies. Annu Rev Psychol, 63, 1-29. doi: 10.1146/annurev-psych-120710-100422

Baddeley, A. D. (2001). Is working memory still working? Am Psychol, 56(11), 851864. doi: 10.1037/0003-066x.56.11.851

Baddeley, A. D., \& Hitch, G. (1974). Working memory Psychology of learning and motivation (Vol. 8, pp. 47-89): Academic press.

Bathelt, J., Gathercole, S. E., Johnson, A., \& Astle, D. E. (2018). Differences in brain morphology and working memory capacity across childhood. Dev Sci, 21(3), e12579. doi: 10.1111/desc. 12579

Burzynska, A. Z., Nagel, I. E., Preuschhof, C., Li, S. C., Lindenberger, U., Backman, L., \& Heekeren, H. R. (2011). Microstructure of frontoparietal connections predicts cortical responsivity and working memory performance. Cereb Cortex, 21(10), 2261-2271. doi: 10.1093/cercor/bhq293

Chai, W. J., Abd Hamid, A. I., \& Abdullah, J. M. (2018). Working Memory From the Psychological and Neurosciences Perspectives: A Review. Front Psychol, 9, 401. doi: 10.3389/fpsyg.2018.00401

Charlton, R. A., Barrick, T. R., Markus, H. S., \& Morris, R. G. (2013). Verbal working and long-term episodic memory associations with white matter microstructure in 
normal aging investigated using tract-based spatial statistics. Psychol Aging, 28(3), 768-777. doi: $10.1037 / \mathrm{a} 0032668$

Chung, S., Fieremans, E., Kucukboyaci, N. E., Wang, X., Morton, C. J., Novikov, D. S., . . Lui, Y. W. (2018). Working Memory And Brain Tissue Microstructure: White Matter Tract Integrity Based On Multi-Shell Diffusion MRI. Sci Rep, 8(1), 3175. doi: $10.1038 / \mathrm{s} 41598-018-21428-4$

Cowan, N. (1988). Evolving conceptions of memory storage, selective attention, and their mutual constraints within the human information-processing system. Psychol Bull, 104(2), 163-191. doi:

Cowan, N. (1999). An Embedded-Processes Model of Working Memory. In A. Miyake \& P. Shah (Eds.), Models of working memory: Mechanisms of active maintenance and executive control (pp. 62-101): Cambridge University Press.

Cowan, N. (2008). What are the differences between long-term, short-term, and working memory? Progress in brain research, 169, 323-338. doi: 10.1016/s00796123(07)00020-9

Cowan, N. (2014). Working Memory Underpins Cognitive Development, Learning, and Education. Educ Psychol Rev, 26(2), 197-223. doi: 10.1007/s10648-013-9246-y

Daneman, M., \& Carpenter, P. A. (1980). Individual differences in working memory and reading. Journal of Verbal Learning and Verbal Behavior, 19(4), 450-466. doi: $10.1016 / \mathrm{s} 0022-5371(80) 90312-6$

Daneman, M., \& Merikle, P. M. (1996). Working memory and language comprehension: A meta-analysis. Psychon Bull Rev, 3(4), 422-433. doi: $10.3758 / \mathrm{BF} 03214546$

Darki, F., \& Klingberg, T. (2015). The role of fronto-parietal and fronto-striatal networks in the development of working memory: a longitudinal study. Cereb Cortex, 25(6), 1587-1595. doi: 10.1093/cercor/bht352 
Ecker, U. K., Lewandowsky, S., Oberauer, K., \& Chee, A. E. (2010). The components of working memory updating: an experimental decomposition and individual differences. $\mathrm{J}$ Exp Psychol Learn Mem Cogn, 36(1), 170-189. doi: 10.1037/a0017891

Ekman, M., Fiebach, C. J., Melzer, C., Tittgemeyer, M., \& Derrfuss, J. (2016). Different Roles of Direct and Indirect Frontoparietal Pathways for Individual Working Memory Capacity. J Neurosci, 36(10), 2894-2903. doi: 10.1523/JNEUROSCI.1376-14.2016

Engle, R. W. (2016). Working Memory Capacity as Executive Attention. Current Directions in Psychological Science, 11(1), 19-23. doi: 10.1111/1467-8721.00160

Fedorova, O. V. (2010). Osnovy eksperimentalnoy psikholingvistiki: rabochaya pamyat i ponimanie rechi [Principles of experimental psycholynguistics: Working memory and language comprehension]. Moscow: Sputnik+.

Gathercole, S. E., Pickering, S. J., Ambridge, B., \& Wearing, H. (2004). The structure of working memory from 4 to 15 years of age. Dev Psychol, 40(2), 177-190. doi: 10.1037/0012-1649.40.2.177

Goldman-Rakic, P. S. (1994). Working memory dysfunction in schizophrenia The Frontal Lobes and Neuropsychiatric Illness (pp. 71-82). Washington, DC.

Hornung, C., Brunner, M., Reuter, R. A. P., \& Martin, R. (2011). Children's working memory: Its structure and relationship to fluid intelligence. Intelligence, 39(4), 210-221. doi: 10.1016/j.intell.2011.03.002

Jenkinson, M., Beckmann, C. F., Behrens, T. E., Woolrich, M. W., \& Smith, S. M. (2012). Fsl. Neuroimage, 62(2), 782-790. doi: 10.1016/j.neuroimage.2011.09.015

Klingberg, T. (2006). Development of a superior frontal-intraparietal network for visuo-spatial working memory. Neuropsychologia, 44(11), 2171-2177. doi: 10.1016/j.neuropsychologia.2005.11.019

Krogsrud, S. K., Fjell, A. M., Tamnes, C. K., Grydeland, H., Due-Tonnessen, P., Bjornerud, A., . . . Walhovd, K. B. (2018). Development of white matter microstructure in relation to verbal and visuospatial working memory-A longitudinal study. PLoS One, 13(4), e0195540. doi: 10.1371/journal.pone.0195540 
Miller, G. A., Galanter, E., \& Pribram, K. H. (1960). Plans and the structure of behavior: Henry Holt and Co.

Miyake, A., Friedman, N. P., Emerson, M. J., Witzki, A. H., Howerter, A., \& Wager, T. D. (2000). The unity and diversity of executive functions and their contributions to complex "Frontal Lobe" tasks: a latent variable analysis. Cogn Psychol, 41(1), 49-100. doi: 10.1006/cogp.1999.0734

Nagy, Z., Westerberg, H., \& Klingberg, T. (2004). Maturation of white matter is associated with the development of cognitive functions during childhood. J Cogn Neurosci, 16(7), 1227-1233. doi: 10.1162/0898929041920441

Oberauer, K. (2002). Access to information in working memory: Exploring the focus of attention. Journal of Experimental Psychology: Learning, Memory, and Cognition, 28(3), 411-421. doi: 10.1037/0278-7393.28.3.411

Oberauer, K., Schulze, R., Wilhelm, O., \& Suss, H. M. (2005). Working memory and intelligence--their correlation and their relation: comment on Ackerman, Beier, and Boyle (2005). Psychol Bull, 131(1), 61-65; author reply 72-65. doi: 10.1037/0033-2909.131.1.61

Oguz, I., Farzinfar, M., Matsui, J., Budin, F., Liu, Z., Gerig, G., . . Styner, M. (2014). DTIPrep: quality control of diffusion-weighted images. Front Neuroinform, 8, 4. doi: 10.3389/fninf.2014.00004

Oldfield, R. C. (1971). The assessment and analysis of handedness: The Edinburgh inventory. Neuropsychologia, 9(1), 97-113. doi: 10.1016/0028-3932(71)90067-4

Olesen, P. J., Nagy, Z., Westerberg, H., \& Klingberg, T. (2003). Combined analysis of DTI and fMRI data reveals a joint maturation of white and grey matter in a fronto-parietal network. Brain Res Cogn Brain Res, 18(1), 48-57. doi: 10.1016/j.cogbrainres.2003.09.003

Ostby, Y., Tamnes, C. K., Fjell, A. M., \& Walhovd, K. B. (2011). Morphometry and connectivity of the fronto-parietal verbal working memory network in development. Neuropsychologia, 49(14), 3854-3862. doi: 10.1016/j.neuropsychologia.2011.10.001 
Sala-Llonch, R., Palacios, E. M., Junque, C., Bargallo, N., \& Vendrell, P. (2015). Functional networks and structural connectivity of visuospatial and visuoperceptual working memory. Front Hum Neurosci, 9, 340. doi: 10.3389/fnhum.2015.00340

Salminen, T., Martensson, J., Schubert, T., \& Kuhn, S. (2016). Increased integrity of white matter pathways after dual n-back training. Neuroimage, 133, 244-250. doi: 10.1016/j.neuroimage.2016.03.028

Sandry, J. (2015). Working memory and memory loss in neurodegenerative disease. Neurodegener Dis Manag, 5(1), 1-4. doi: 10.2217/nmt.14.51

Schmiedek, F., Lovden, M., \& Lindenberger, U. (2014). A task is a task is a task: putting complex span, n-back, and other working memory indicators in psychometric context. Front Psychol, 5, 1475. doi: 10.3389/fpsyg.2014.01475

Schulze, E. T., Geary, E. K., Susmaras, T. M., Paliga, J. T., Maki, P. M., \& Little, D. M. (2011). Anatomical correlates of age-related working memory declines. J Aging Res, 2011, 606871. doi: 10.4061/2011/606871

Short, S. J., Elison, J. T., Goldman, B. D., Styner, M., Gu, H., Connelly, M., . . . Gilmore, J. H. (2013). Associations between white matter microstructure and infants' working memory. Neuroimage, 64, 156-166. doi: 10.1016/j.neuroimage.2012.09.021

Smith, S. M., Jenkinson, M., Johansen-Berg, H., Rueckert, D., Nichols, T. E., Mackay, C. E., . . Behrens, T. E. (2006). Tract-based spatial statistics: voxelwise analysis of multi-subject diffusion data. Neuroimage, 31(4), 1487-1505. doi:

10.1016/j.neuroimage.2006.02.024

Süß, H.-M., Oberauer, K., Wittmann, W. W., Wilhelm, O., \& Schulze, R. (2002). Working-memory capacity explains reasoning ability — and a little bit more. Intelligence, 30(3), 261-288. doi: 10.1016/s0160-2896(01)00100-3

Takahashi, M., Iwamoto, K., Fukatsu, H., Naganawa, S., Iidaka, T., \& Ozaki, N. (2010). White matter microstructure of the cingulum and cerebellar peduncle is related to sustained attention and working memory: a diffusion tensor imaging study. Neurosci Lett, 477(2), 72-76. doi: 10.1016/j.neulet.2010.04.031 
Takeuchi, H., Sekiguchi, A., Taki, Y., Yokoyama, S., Yomogida, Y., Komuro, N., . . Kawashima, R. (2010). Training of working memory impacts structural connectivity. J Neurosci, 30(9), 3297-3303. doi: 10.1523/JNEUROSCI.4611-09.2010

Takeuchi, H., Taki, Y., Sassa, Y., Hashizume, H., Sekiguchi, A., Fukushima, A., \& Kawashima, R. (2011). Verbal working memory performance correlates with regional white matter structures in the frontoparietal regions. Neuropsychologia, 49(12), 3466-3473. doi: 10.1016/j.neuropsychologia.2011.08.022

Vestergaard, M., Madsen, K. S., Baare, W. F., Skimminge, A., Ejersbo, L. R., Ramsoy, T. Z., . . Jernigan, T. L. (2011). White matter microstructure in superior longitudinal fasciculus associated with spatial working memory performance in children. J Cogn Neurosci, 23(9), 2135-2146. doi: 10.1162/jocn.2010.21592

Wilhelm, O., Hildebrandt, A., \& Oberauer, K. (2013). What is working memory capacity, and how can we measure it? Front Psychol, 4, 433. doi: 10.3389/fpsyg.2013.00433

Winkler, A. M., Ridgway, G. R., Webster, M. A., Smith, S. M., \& Nichols, T. E. (2014). Permutation inference for the general linear model. Neuroimage, 92, 381-397. doi: 10.1016/j.neuroimage.2014.01.060

Zahr, N. M., Rohlfing, T., Pfefferbaum, A., \& Sullivan, E. V. (2009). Problem solving, working memory, and motor correlates of association and commissural fiber bundles in normal aging: a quantitative fiber tracking study. Neuroimage, 44(3), 1050-1062. doi: 10.1016/j.neuroimage.2008.09.046 